References

[1] F. E. Browder, Functional analysis and partial differential equations $I$, MathAnn. 138 (1959), pp. 55-79.

[2] A. K. Chilana, Relatively continuous operators and some perturbation results, J. London Math. Soc. II. Ser. 2 (1970), pp. 225-231.

[3] K. H. Förster, Relativ co-stetige Operatoren in normierten Räumen, Arch. Math. 25 (1974), pp. 639-645.

[4] J. Horvath, Topological vector spaces and distributions I, Addison-Wesley Reading - Palo Alto-Isondon-Don Mils 1966.

[5] G. Köthe, Topologische lineare Räume I, Springer, Berlin-Göttingen-Heidelberg 1960 .

[6] - General linear transformations of locally convex spaces, Math. Ann. 159 (1965) pp. 309-328.

[7] J. Laustaunau, On the state diagram of a linear operator and its adjoint in locally convex spaces, II, Math. Ann. 176 (1968), pp. 121-128.

[8] A. Pietsch, Nukleare lokalkonvexe Räume, 2. Aufl. Akademie-Verlag, Berlin 1969.

\section{On local ergodic theorems for positive semigroups}

\section{by}

\author{
RYOTARO SATO (Sakado)
}

Abstract. For a one-parameter semigroup $\Gamma=\left\{T_{t} ; t>0\right\}$ of positive linear operators on $L_{1}$ of a $\sigma$-finite measure space which is assumed to be strongly integrable over every finite interval, the following local ergodic theorem is proved: If $0 \leqslant f \in L_{1}$ and $\|f\|_{1}>0$ imply sup $\left\|T_{t} f\right\|_{1}>0$, then for any $f \in L_{1}$ the limit

$$
t>0
$$

$$
\lim _{b \rightarrow+0} \frac{1}{b} \int_{0}^{b} T_{t} f d t
$$

exists and is finte almost everywhere. Under the assumption that $\Gamma$ is locally bounded, i.e., sup $\left\|T_{t}\right\|_{1}<\infty$, a necessary and sufficient condition is given for the possibility $0<t<1$

of completing $\Gamma$ to a strongly continuous semigroup on $[0, \infty)$. A local ergodic theorem for the adjoint semigroup $\Gamma^{*}=\left\{T_{t}^{*} ; t>0\right\}$ of $\Gamma$ is also considered.

1. Introduction and theorems. Let $(X, \mathscr{F}, \mu)$ be a $\sigma$-finite measure space with positive measure $\mu$, and let $L_{p}(X)=L_{p}(X, \mathscr{F}, \mu), 1 \leqslant p \leqslant \infty$, denote the (complex) Banach spaces defined as usual with respect to $(X, \mathscr{F}, \mu)$. All sets and functions introduced below are assumed to be measurable; all relations are assumed to hold modulo sets of measure zero. If $A$ is a subset of $X$, then $1_{A}$ is the indicator function of $A$ and $L_{p}(A)$ denotes the Banach space of all $L_{p}(X)$-functions that vanish on $X-A$. Also, $L_{p}^{+}(A)$ denotes the positive cone of $L_{p}(A)$ consisting of nonnegative $L_{p}(A)$-functions. A linear operator $T$ on $L_{p}(X)$ is called positive if $T\left(L_{p}^{+}(X)\right)$ $\subset L_{p}^{+}(X)$, and a contraction if $\|T\|_{p} \leqslant 1$. It is well known that if $T$ is positive, then $\|T\|_{p}<\infty$. The adjoint of $T$ is denoted by $T^{*}$.

Let $\Gamma=\left\{T_{t} ; t>0\right\}$ be a one-parameter semigroup of positive linear operators on $L_{1}(X)$, i.e., all the $T_{t}$ are positive linear operators on $L_{1}(X)$ and $T_{t} T_{t^{\prime}}=T_{t+t^{\prime}}$ for all $t, t^{\prime}>0$. In this paper we assume that $\Gamma$ is strongly integrable over every finite interval. This means that for each $f \in L_{1}(X)$ the vector-valued function $t \rightarrow T_{t} f$ is integrable with respect to Lebesgue measure on every finite interval. It then follows from Lemma VIII.1.3 of [4] that $\Gamma$ is strongly continuous on $(0, \infty)$, i.e., for each $f \in L_{1}(X)$ and each $s>0$ we have $\lim \left\|T_{t} f-T_{s} f\right\|_{1}=0$. Hence, by an approximation argument (cf. [13], Section 4), we observe that for each $f \in L_{1}(X)$ there exists 
a scalar function $T_{t} f(x)$, measurable with respect to the product of the Lebesgue measurable subsets of $(0, \infty)$ and $\mathscr{F}$, such that, for each fixed $t>0, T_{t} f(x)$, as a function of $x$, belongs to the equivalence class of $T_{t} f$. From this and Fubini's theorem it now follows that there exists a subset $E(f)$ of $X$ with $\mu(E(f))=0$, dependent on $f$ but independent of $t$, such that if $x \notin E(f)$, then the function $t \rightarrow T_{t} f(x)$ is integrable with respect to Lebesgue measure on every finite interval $(a, b) \subset(0, \infty)$ and the integral $\int_{a}^{b} T_{t} f(x) d t$, as a function of $x$, belongs to the equivalence class of $\int_{a}^{b} T_{t} f d t$. $\Gamma$ is called locally bounded if $\sup _{0<t<1}\left\|T_{t}\right\|_{1}<\infty$, and bounded if $\sup \left\|T_{t}\right\|_{1}<\infty$. Next, let $\Gamma^{*}=\left\{T_{t}^{*} ; t>0\right\}$ denote the adjoint semigroup of $\Gamma$. Therefore, $\Gamma^{*}$ acts on $L_{\infty}(X)$, and $\left\langle u, T_{t}^{*} f\right\rangle=\left\langle T_{t} u, f\right\rangle$ for all $u \in L_{1}(X)$, $f \in L_{\infty}(X)$ and $t>0$. For $0 \leqslant a<b<\infty$ and $f \in L_{\infty}(X)$, we let

$$
\int_{a}^{b} T_{t}^{*} f d t=\left(\int_{a}^{b} T_{t} d t\right)^{*} f
$$

Therefore,

$$
\int_{a}^{b} T_{t}^{*} f d t \in L_{\infty}(X) \quad \text { and } \quad\left\langle u, \int_{a}^{b} T_{t}^{*} f d t\right\rangle=\left\langle\int_{a}^{b} T_{t} u d t, f\right\rangle
$$

for all $u \in L_{1}(X)$. A slight modification of the proof of Theorem 1.1 of Lin [11] implies that for each $f \in L_{\infty}(X)$ there exists a scalar function $T_{t}^{*} f(x)$, measurable with respect to the product of the Lebesgue measirable subsets of $(0, \infty)$ and $\mathscr{F}$, and a subset $E(f)$ of $X$ with $\mu(E(f))=0$, dependent on $f$ but independent of $t$, such that if $x \notin E(f)$, then the function $t \rightarrow T_{t}^{*} f(x)$ is integrable with respect to Lebesgue measure on every finite interval $(a, b) \subset(0, \infty)$ and the integral $\int_{a}^{b} T_{t}^{*} f(x) d t$, as a function of $x$, belongs to the equivalence class of $\int_{a}^{b} T_{i}^{*} f d t$.

Under the additional assumption that all the $T_{t}$ are contractions on $L_{1}(X)$, Akcoglu and Chacon [1] proved that for any $f \in L_{1}(X)$ the following local erogodic limit

$$
\lim _{b \rightarrow+0} \frac{1}{b} \int_{0}^{b} T_{t} f(x) d t
$$

exists and is finite a.e. on $X . \cdot$ Related results in this direction have been obtained by Krengel [7], Ornstein [13], Fong and Sucheston [5], Kubokawa [8], [9], [10], Baxter and Chacon [2], McGrath [12], the author [15], [16], and by others.

In this paper we apply Akcoglu-Chacon's local ergodic theorem to obtain the following results:
THEOREM 1. Let $\Gamma=\left\{T_{t} ; t>0\right\}$ be a one-parameter semigroup of positive linear operators on $L_{1}(X)$, strongly integrable over every finite interval. Then the space $X$ decomposes into two sets $P$ and $N$ such that

(i) for all $f \in L_{1}(N)$ and $t>0$, we have $\left\|T_{t} f\right\|_{1}=0$,

(ii) for all $f \in L_{1}^{+}(P)$, with $\|f\|_{1}>0$, we have $\sup _{t>0}\left\|\left(T_{t} f\right) 1_{P}\right\|_{1}>0$.

For any $f \in L_{1}(X)$ the limit (1) exists and is finite a.e. on $P$.

The next theorem shows that, on $N$, the almost everywhere existence of the limit (1) need not hold in general for all $f \in L_{1}(X)$.

THEOREM 2. For any $\varepsilon>0$ there exists a finite measure space $(X, \mathscr{F}, \mu)$, and a one-parameter semigroup $\Gamma=\left\{T_{t} ; t \geqslant 0\right\}$ of positive linear operators on $L_{1}(X)$, strongly continuous on $[0, \infty)$, such that

(i) for all $t \geqslant 0$, we have $T_{t} 1=1$ and $\left\|T_{t}\right\|_{1}=1+\varepsilon$,

(ii) $\mu(N)>0$, where $X=P+N$ is the decomposition given in Theorem 1 ,

(iii) for some $f \in L_{1}^{+}(X)$, the limit (1) does not exist a.e. on $N$.

THeOREM 3. Let $\Gamma=\left\{T_{t} ; t>0\right\}$ be a one-parameter semigroup of positive linear operators on $L_{1}(X)$, strongly integrable over every finite interval. Then the spaee $X$ decomposes into two sets $C$ and $D$ such that

(i) for all $f \in L_{1}(X)$ and $t>0$, we have $T_{t} f \in L_{1}(C)$,

(ii) for all $f \in L_{1}^{+}(C)$, with $f>0$ a.e. on $C$, we have

$$
C=\bigcup_{n=1}^{\infty}\left\{x \in X: T_{1 / n} f(x)>0\right\} \text {. }
$$

Assume, in addition, that $\Gamma$ is locally bounded. Then $T_{t}$ converges strongly as $t \rightarrow+0$ if and only if there exists a function $g \in L_{1}^{+}(D), g>0$ a.e. on $D$, and a decreasing sequence $\left(b_{n}\right)$ of positive reals, with $\lim b_{n}=0$, such that the set

$$
\left\{\frac{1}{b_{n}} \int_{0}^{b_{n}} T_{t} g d t: n \geqslant 1\right\}
$$

is weakly sequentially compact in $L_{1}(X)$.

THEOREM 4. Let $\Gamma=\left\{T_{i} ; t>0\right\}$ be a one-parameter semigroup of positive linear operators on $L_{1}(X)$, strongly integrable over every finite interval. Assume that $X=C$ in Theorem 3. Then for each $f \in L_{\infty}(X)$ there exists a scalar function $T_{t}^{*} f(x)$, measurable with respect to the product of the Lebesgue measurable subsets of $(0, \infty)$ and $\mathscr{F}$, such that for each fixed $t>0, T_{i}^{*} f(x)$, as a function of $x$, belongs to the equivalence class of $T_{t}^{*} f$; moreover, the follow- 
ing local ergodic limit

$$
\lim _{b \rightarrow+0} \frac{1}{b} \int_{0}^{b} T_{t}^{*} f(x) d t
$$

exists and is finite a.e. on $X$.

Theorem 1 contains a local ergodic theorem of Kubokawa [8] as a special case, since $X=P$ for every one-parameter semigroup $\Gamma=\left\{T_{t}\right.$; $t>0\}$ of positive linear operators on $L_{1}(X)$ which is strongly continuous on $(0, \infty)$ and satisfies strong- $\lim T_{t}=I$ (the identity operator). Theorem 3 generalizes Theorem 4.1 of $\stackrel{t \rightarrow+0}{A k \operatorname{kglu}}$ and Chacon [1]; their argument is due to the almost everywhere existence of the limit (1) and Lebesgue's convergence theorem, therefore the additional assumption that all the $T_{t}$ are contractions on $L_{1}(X)$ cannot be weakened in the argument (cf. Theorem 2). Theorem 4, together with Theorems 1 and 3 , generalizes Theorem 1 of Krengel [6], Theorem 7.2 of Lin [11], and a part of Corollary 2 of the author [14].

2. Proof of Theorem 1. Let $\mathscr{A}=\left\{A \in \mathscr{F}: f \in L_{1}(A)\right.$ implies $\left\|T_{t} f\right\|_{1}=0$ for all $t>0\}$. Since $A, B \in \mathscr{A}$ implies $A \cup B \in \mathscr{A}$, and since $(X, \mathscr{F}, \mu)$ is a $\sigma$-finite measure space, there exists a set $N \in \mathscr{A}$ with $A \subset N$ for all $A \in \mathscr{A}$. To prove (ii), let $P=X-N$ and let $f \in L_{1}^{+}(P)$ be a nonzero function. By the definition of $N$, we have $\left\|T_{t} f\right\|_{1}>0$ for some $t>0$, therefore by (i)

$$
\left\|T_{t / 2}\left(\left(T_{t / 2} f\right) 1_{P}\right)\right\|_{1}=\left\|T_{t / 2}\left(T_{t / 2} f\right)\right\|_{1}=\left\|T_{t} f\right\|_{1}>0 .
$$

To prove the remainder of the theorem we may and will assume without loss of generality that $X=P$. Let $a>0$ be a constant satisfying $e^{-a}\left\|T_{1}\right\|_{1}<1$. Then it follows that for each $f \in L_{1}(X)$ the vector-valued function $t \rightarrow e^{-a t} T_{t} f$ is integrable with respect to Lebesgue measure on $(0, \infty)$. Therefore we can define a positive linear operator $R$ on $L_{1}(X)$ by the relation

$$
R f=\int_{0}^{\infty} e^{-a t} T_{t} f d t \quad\left(f \in L_{1}(X)\right)
$$

Let $u=R^{*} 1 \in L_{\infty}^{+}(X)$. Then for any $f \in L_{1}^{+}(X)$, with $\|f\|_{1}>0$, we have

$$
\begin{aligned}
\langle f, u\rangle=\langle R f, 1\rangle & =\left\langle\int_{0}^{\infty} e^{-a t} T_{t} f d t, 1\right\rangle \\
& =\int_{0}^{\infty} e^{-a t}\left\langle T_{t} f, 1\right\rangle d t>0,
\end{aligned}
$$

since $\left\langle T_{t} f, 1\right\rangle$ is a nonnegative nonzero continuous function on $(0, \infty)$. It follows that $u>0$ a.e. on $X$. By a similar calculation, we have $T_{t}^{*} u \leqslant e^{a t} u$ for all $t>0$. We now define a one-parameter semigroup $\Delta=\left\{S_{t} ; t>0\right\}$ of positive linear contractions on $L_{1}(X)$ which is strongly continuous on $(0, \infty)$ as follows:

For $t>0$ and $u f \in L_{1}(X)$, where $f \in L_{1}(X)$, let

$$
S_{t}(u f)=e^{-a t} u\left(T_{t} f\right) \text {. }
$$

Since

$$
\left\|S_{t}(u f)\right\|_{1}=\int_{X} e^{-a t} u\left(T_{t} f\right) d \mu=\cdot \int_{X}^{\bar{I}} e^{-a t}\left(T_{t}^{*} u\right) f d \mu \leqslant \int_{X} u f d \mu
$$

for all $f \in L_{1}^{+}(X)$, and since $\left\{u f: f \in L_{1}(X)\right\}$ is a dense subspace of $L_{1}(X)$ in the norm topology, $S_{t}$ may be considered to be a positive linear contraction on $L_{1}(X)$. By an approximation argument we observe that $S_{t} S_{t^{\prime}}=S_{t+t^{\prime}}$ for all $t, t^{\prime}>0$, and that for each fixed $f \in L_{1}(X)$, the mapping $t \rightarrow S_{t} f$ is strongly continuous on $(0, \infty)$. Therefore we may apply Akcoglu-Chacon's local ergodic theorem to $\Delta$ to infer that for any $f \in L_{1}(X)$ the limit

$$
\lim _{b \rightarrow+0} \frac{1}{b} \int_{0}^{b} S_{t}(u f)(x) d t
$$

exists and is finite a.e. on $X$. This proves that for any $f \in L_{1}(X)$ the limit (1) exists and is finite a.e. on $X$, since $T_{t} f=e^{a t} S_{t}(u f) / u$ for all $t>0$ and $\lim e^{a t}=1$.

$t \rightarrow+0$

The proof is complete.

Remark 1. If all the $T_{t}$ are contractions on $L_{1}(X)$, then $T_{t}\left(L_{1}(P)\right)$ $\subset L_{1}(P)$.

To see this, let $g \in L_{1}^{+}(P), g>0$ a.e. on $P$, and $t>0$. Define $h_{1}=\left(T_{t} g\right) 1_{P}$ and $h_{2}=\left(T_{t} g\right) 1_{N}$. Then, since $\Gamma$ is strongly continuous on $(0, \infty)$, we have

$$
h_{1}+h_{2}=\lim _{s \rightarrow+0} T_{s}\left(h_{1}+h_{2}\right)=\lim _{s \rightarrow+0} T_{s} h_{1}
$$

where the second equality follows from (i) of Theorem 1 . Since $\left\|T_{s}\right\|_{1} \leqslant 1$ for all $s>0$, we conclude that $\left\|h_{2}\right\|_{1}=0$, and this completes the proof of Remark 1 .

3. Proof of Theorem 2. To prove the theorem, we give the following example:

Let $L_{1}=L_{1}(0,1]$ (with Lebesgue measure) and let, for each $t \geqslant 0$,

$$
S_{t} f(x)=f([x+t]) \quad\left(f \in L_{1}, 0<x \leqslant 1\right),
$$

where $[x+t]=x+t$ if $x+t \leqslant 1$ and $[x+t]=x+t-n$ if $n<x+t \leqslant n+1$. Then it may be readily seen that $\Delta=\left\{S_{i} ; t \geqslant 0\right\}$ is a one-parameter semi- 
group of positive linear contractions on $L_{1}$ and strongly continuous on $[0, \infty)$. Let $\left(\alpha_{n}\right)$ be a sequence of positive reals satisfying

$$
a_{n}>1 \text { for all } n, \quad \text { and } \quad \sum_{n=1}^{\infty}\left(1 / 2^{n}\right) \log \alpha_{n}=\infty .
$$

Choose a sequence $\left(\beta_{n}\right)$ of positive reals satisfying

$$
\sum_{n=1}^{\infty} \beta_{n}<1, \quad \text { and }\left(1 / \beta_{n}\right) \sum_{i>n} \beta_{i}<1 / \alpha_{n} \text { for all } n .
$$

We then define

$$
d_{0}=0, \quad d_{n}=\sum_{i=1}^{n} \beta_{i}, \quad d=\sum_{i=1}^{\infty} \beta_{i}, \quad a_{n}=\left(2^{n} \beta_{n}\right)^{-1},
$$

and a function $g$ on $(0,1]$ by the relation

$$
g(x)=\left\{\begin{array}{lll}
a_{n} & \text { if } & d_{n-1}<x \leqslant d_{n}, \\
0 & \text { if } & d \leqslant x \leqslant 1 .
\end{array}\right.
$$

It is obvious that $g \in L_{1}^{+}$. But, an elementary calculation shows that if we let

$$
g^{*}(x)=\sup _{0<b<1} \frac{1}{b} \int_{0}^{b} S_{t} g(x) d t
$$

then $g^{*} \notin L_{1}$. Therefore there exists a sequence $\left(b_{n}\right)$ of positive reals; with $\lim b_{n}=0$, such that if we let

$$
g_{\infty}^{*}(x)=\sup _{n} \frac{1}{b_{n}} \int_{0}^{b_{n}} S_{t} g(x) d t
$$

then $g_{\infty}^{*} \notin L_{1}$. Since

$$
\lim _{n} \frac{1}{b_{n}} \int_{0}^{b_{n}} S_{t} g(x) d t=g(x) \quad \text { a.e. on }(0,1]
$$

by Akcoglu-Chacon's local ergodic theorem and since

$$
\lim _{n}\left\|\frac{1}{b_{n}} \int_{0}^{b_{n}} S_{t} g d t-g\right\|_{1}=0
$$

it now follows from Theorem 4.3 of Derriennic and Lin [3] that there exists a sub- $\sigma$-field $\mathscr{B}$ of the Lebesgue measurable subsets of $(0,1]$ such that $\lim _{n} E\left(\frac{1}{b_{n}} \int_{0}^{b_{n}} S_{t} g d t \mid \mathscr{B}\right)(x)$ does not exist a.e. on $(0,1]$, where for any $f \in L_{1}, E(f \mid \mathscr{B})$ denotes the conditional expectation of $f$ with respect to $\mathscr{B}$.

Let $\varepsilon>0$ be an arbitrary but fixed number. For each $t \geqslant 0$, define an operator $T_{t}$ on $L_{1}(0,1+\varepsilon]$ by the relation

$$
T_{t} f(x)=\left\{\begin{array}{lll}
S_{t}\left(f I_{(0,1]}\right)(x) & \text { if } & 0<x \leqslant 1 \\
E\left(S_{t}\left(f I_{(0,1]}\right) \mid \mathscr{B}\right)\left(\frac{x-1}{\varepsilon}\right) & \text { if } & 1<x \leqslant 1+\varepsilon .
\end{array}\right.
$$

Then it is easily seen that $\Gamma=\left\{T_{t} ; t \geqslant 0\right\}$ is a one-parameter semigroup of positive linear operators on $L_{1}(0,1+\varepsilon]$ and strongly continuous on $[0, \infty)$; moreover for any $t \geqslant 0$ we have $T_{t} 1=1$ and $\left\|T_{i}\right\|_{1}=1+\varepsilon$. It is clear that $P=(0,1]$ and $N=(1,1+\varepsilon]$. Let $h \in L_{1}^{+}(0,1+\varepsilon]$ be such that $h=g$ a.e. on $P$. Then for each $n \geqslant 1$ we have, a.e. on $N$,

$$
\begin{aligned}
\frac{1}{b_{n}} \int_{0}^{b_{n}} T_{t} h(x) d t & =\left(\frac{1}{b_{n}} \int_{0}^{b_{n}} E\left(S_{t} g \mid \mathscr{B}\right) d t\right)\left(\frac{x-1}{\varepsilon}\right) \\
& =E\left(\frac{1}{b_{n}} \int_{0}^{b_{n}} S_{t} g d t \mid \mathscr{B}\right)\left(\frac{x-1}{\varepsilon}\right) .
\end{aligned}
$$

Therefore, $\lim _{n} \frac{1}{b_{n}} \int_{0}^{b_{n}} T_{t} h(x) d t$ (and hence $\lim _{b \rightarrow+0} \frac{1}{b} \int_{0}^{b} T_{t} h(x) d t$ ) does not exist a.e. on $N$.

This completes the proof of Theorem 2.

4. Proof of Theorem 3. Let $h \in L_{1}^{+}(X), h>0$ a.e. on $X$, and let

$$
C=\bigcup_{n=1}^{\infty}\left\{x \in X: T_{1 / n} h(x)>0\right\} .
$$

Then, by an approximation argument, we observe that $T_{i} h \in L_{1}(C)$ for all $t>0$, and that $T_{t} f \in L_{1}(C)$ for all $f \in L_{1}^{+}(X)$ and $t>0$. Thus, (i) is proved.

To prove (ii), let $f \in L_{1}^{+}(C), f>0$ a.e. on $C$. Then, by (i) and an approximation argument, $\left\{x \in X: T_{1 / n} h(x)>0\right\}=\left\{x \in X: T_{1 / 2 n}\left(T_{1 / 2 n} h\right)(x)>0\right\}$ $\subset\left\{x \in X: T_{1 / 2 n} f(x)>0\right\}$ for all $n \geqslant 1$. Hence (ii) follows.

To prove the remainder of the theorem, we assume that $T$ is locally bounded. Then, letting $f_{1}=\int_{0}^{1} T_{t} f d t$ for any $f \in L_{1}^{+}(C)$, with $f>0$ a.e. on $C$, we have that $\lim \left\|T_{t} f_{1}-f_{1}\right\|_{1}=0$ and, by (ii), that $f_{1}>0$ a.e. on $G$. 
Suppose there exists a function $g \in L_{1}^{+}(D), g>0$ a.e. on $D$, and a decreasing sequence $\left(b_{n}\right)$ of positive reals, with $\lim b_{n}=0$, such that the set

$$
\left\{\frac{1}{b_{n}} \int_{0}^{b_{n}} T_{t} g d t: n \geqslant 1\right\}
$$

is weakly sequentially compact in $L_{1}(X)$. Write $h=f_{1}+g$. Then $h>0$ a.e. on $X$, and the set

$$
\left\{\frac{1}{b_{n}} \int_{0}^{b_{n}} T_{t} h d t: n \geqslant 1\right\}
$$

is again weakly sequentially compact in $L_{1}(X)$. Since the local boundedness of $T$ implies

$$
\sup _{n}\left\|\frac{1}{b_{n}} \int_{0}^{b_{n}} T_{t} d t\right\|_{1}<\infty,
$$

it follows from Lemma II.3.30 of [4] that for all $f \in L_{1}(X)$ the set

$$
\left\{\frac{1}{b_{n}} \int_{0}^{b_{n}} T_{t} f d t: n \geqslant 1\right\}
$$

is weakly sequentially compact in $L_{1}(X)$.

To prove the strong convergence of $T_{t}$ as $t \rightarrow+0$, let $f \in L_{1}(X)$ be an arbitrary but fixed function. Since $\Gamma$ is strongly continuous on $(0, \infty)$, there exists a closed separable subspace $H$ of $L_{1}(X)$, with $f \in H$, such that $T_{t}(H) \subset H$ for all $t>0$. Let $\left\{f_{n}: n \geqslant 1\right\}$ be a countable dense subset of $H$. Then, by Cantor's diagonal method, we can find a subsequence $\left(e_{n}\right)$ of $\left(b_{n}\right)$ such that weak- $\lim _{n} \int_{0}^{c_{n}} T_{t} f_{i} d t$ exists for all the $f_{i}, i \geqslant 1$. It now follows from an approximation argument that

$$
\text { weak- } \lim _{n} \frac{1}{c_{n}} \int_{0}^{c_{n}} T_{t} g d t=T_{0} g
$$

exists for all $g \in H$. Since $H$ is a closed subspace of $L_{1}(X), T_{0} g \in H$ for all $g \in H$. It is easily seen that, on $H, T_{t} T_{t^{\prime}}=T_{t+t^{\prime}}$ for all $t, t^{\prime} \geqslant 0$. Thus $\Delta=\left\{T_{t} ; t \geqslant 0\right\}$ may be considered to be a one-parameter semigroup of linear operators on $H$, and it may be readily seen that $T_{0} f$ belongs to the norm-closure of the subspace $\bigcup_{t>0} T_{t}(H)$. Since $\Delta$ is strongly continuous on $(0, \infty)$ and locally bounded, it follows that

$$
\lim _{t \rightarrow+0}\left\|T_{t} f-T_{0} f\right\|_{1}=\lim _{t \rightarrow+0}\left\|T_{t}\left(T_{0} f\right)-T_{0} f\right\|_{1}=0
$$

Hence $T_{t}$ eonverges strongly as $t \rightarrow+0$.
Conversely, suppose that $T_{t}$ converges strongly as $t \rightarrow+0$. Then for any $g \in L_{1}(X)$, strong-lim $\frac{1}{b \rightarrow+0} \int_{0}^{b} T_{t} g d t$ exists, therefore the condition is obviously necessary.

The proof is complete.

5. Proof of Theorem 4. Fix a function $u \in L_{1}^{+}(X)$, with $u>0$ a.e. on $X$, and let

$$
h=\int_{0}^{\infty} e^{-a t} T_{t} u d t\left(\in L_{1}^{+}(X)\right),
$$

where $a>0$ is a constant satisfying $e^{-a}\left\|T_{1}\right\|_{1} \leqslant 1$. Then, by Theorem 3 , we have $h>0$ a.e. on $X$. Therefore, for any $t>0$ and $f \in L_{1}(X, \mathscr{F}, h d \mu)$, we can define

$$
S_{t} f=(1 / h) T_{t}(f h)
$$

Since the mapping $f \rightarrow f h$ is a linear isometry from $L_{1}(X, \mathscr{F}, h d \mu)$ onto $L_{1}(X, \mathscr{F}, \mu)$, it follows that $\Delta=\left\{S_{t} ; t>0\right\}$ is a one-parameter semigroup of positive linear operators on $L_{1}(X, F, h d \mu)$ and strongly integrable over every finite interval. It is easily seen that $\delta_{t}^{*}=T_{t}^{*}$ for all $t>0$ and that $S_{t} 1 \leqslant e^{a t}$ for all $t>0$. Therefore we may and will assume from the first, without loss of generality, that $(X, \mathscr{F}, \mu)$ is a finite measure space and that $\left\|T_{t}\right\|_{\infty} \leqslant e^{a t}$ for all $t>0$. Then, by the Riesz convexity theorem and an approximation argument, $\Gamma$ may be considered to be a one-parameter semigroup of bounded linear operators on $L_{2}(X)$ which is strongly continuous on $(0, \infty)$. Since $L_{2}(X)$ is a Hilbert space and the adjoint semigroup $\Gamma^{*}$ on $L_{2}(X)$ is weakly continuous on $(0, \infty)$, it follows that $\Gamma^{*}$ on $L_{2}(X)$ is strongly continuous on $(0, \infty)$. Hence, again by an approximation argument, we observe that $\Gamma^{*}$ is a one-parameter semigroup of positive linear operators on $L_{1}(X)$ and strongly continuous on $(0, \infty)$. Moreover, $\left\|T_{t}^{*}\right\|_{1}=\left\|T_{t}\right\|_{\infty} \leqslant e^{a t}$ for all $t>0$ implies that $\Gamma^{*}$ on $L_{1}(X)$ is strongly integrable over every finite interval. Therefore, for each $f \in L_{\infty}(X)$ ( $\subset L_{1}(X)$ ), there exists a scalar function $T_{t}^{*} f(x)$, measurable with respect to the product of the Lebesgue measurable subsets of $(0, \infty)$ and $\mathscr{F}$, such that for each fixed $t>0, T_{t}^{*} f(x)$, as a function of $x$, belongs to the equivalence class of $T_{t}^{*} f$.

To prove the remainder of the theorem, we consider the one-parameter semigroup $\left\{e^{-a t} T_{t}^{*} ; t>0\right\}$ of positive linear contractions on $L_{1}(X)$. Since this semigroup is strongly continuous on $(0, \infty)$, we can apply Akcoglu-Chacon's local ergodic theorem to infer that for any $f \in L_{\infty}(X)$ the limit

$$
\lim _{b \rightarrow+0} \frac{1}{b} \int_{0}^{b} e^{-a t} T_{t}^{*} f(x) d t
$$


exists and is finite a.e. on $X$, and hence the limit (2) exists and is finite a.e. on $X$ for all $f \in L_{\infty}(X)$.

The proof is complete.

6. An extension of Theorem 1 . In this section we assume that $\Gamma=\left\{T_{t} ; t>0\right\}$ is a one-parameter semigroup of positive linear operators on $L_{p}(X)$ for some fixed $p$, with $1 \leqslant p<\infty$, and strongly integrable over every finite interval. Then, as in Section 1 , for each $f \in L_{p}(X)$ there exists a scalar function $T_{t} f(x)$, measurable with respect to the product of the Lebesgue measurable subsets of $(0, \infty)$ and $\mathscr{F}$, such that for each fixed $t>0, T_{t} f(x)$, as a function of $x$, belongs to the equivalence class of $T_{t} f_{\text {; }}$ And there exists a subset $E(f)$ of $\bar{X}$ with $\mu(E(f))=0$, dependent on $f$ but independent of $t$, such that if $x \notin E(f)$, then the function $t_{\mapsto \rightarrow} T_{t} f(x)$ is integrable with respect to Lebesgue measure on every finite interval $(a, b) \subset(\dot{0}, \infty)$ and the integral $\int_{a}^{b} T_{t} f(x) d t$, as a function of $x$, belongs to the equivalence class of $\int_{a}^{b} T_{t} f d t$.

Slightly modifying the proof of Theorem 1, we can prove the following extension of Theorem 1:

THEOREM 5. Let $\Gamma=\left\{T_{t} ; t>0\right\}$ be a one-parameter semigroup of positive linear operators on $L_{p}(X)$ for some fixed $p$, with $1 \leqslant p<\infty$, and strongly integrable over every finite interval. Then the space $X$ decomposes into two sets $P$ and $N$ such that

(i) for all $f \in L_{p}(N)$ and $t>0$, we have $\left\|T_{t} f\right\|_{p}=0$,

(ii) for all $f \in L_{p}^{+}(P)$, with $\|f\|_{p}>0$, we have $\sup _{t>0}\left\|\left(\boldsymbol{T}_{t} f\right) 1_{P}\right\|_{p}>0$.

For any $f \in L_{p}(X)$ the limit (1) exists and is finite a.e. on $P$.

We note that this theorem generalizes Kubokawa's local ergodic theorem ([9], Theorem 1).

Remark 2. Let $1<p<\infty$, and let $\Gamma=\left\{T_{t} ; t>0\right\}$ be a one-parameter semigroup of bounded linear operators on $L_{p}(X)$. If $\Gamma$ is strongly continuous on $(0, \infty)$ and locally bounded, then $T_{t}$ converges strongly as $t \rightarrow+0$.

This follows from Lemma 1 of the author [15], since $L_{p}(\boldsymbol{X})$, with $1<p<\infty$, is a reflexive Banach space.

\section{Reference}

[1] M. A. Akeoglu and R. V. Chacon, A loaal ratio theorem, Canad. J. Math. 22 (1970), pp. 545-552.

[2] J. R. Baxter and R. V. Chacon, A lacal ergodic theorem on $L_{p}$, ibid. 26 (1974), pp. 1206-1216.

[3] Y. Derriennic and M. Lin, On invariant measures and ergodic theorems for positive operators, J. Functional Analysis 13 (1973), pp. 252-267.
[4] N. D unford and J. T. Schwartz, Iinear operators, Part I: General theory, New York 1958.

[5] H. F ong and L. Sucheston, On the ratio ergodic theorem for semi-groups, Pacific J. Math. 39 (1971), pp. 659-667.

[6] U. Krengel, A necessary and sufficient condition for the validity of the local ergodic theorem, in: Probability and Information Theory (Proc. Internat. Sympos., McMaster Univ., Hamilton, Ont. 1968), pp. 170-177, Berlin-Heidelberg-New York 1969.

[7] - A local ergodic theorem, Invent. Math. 6 (1969), pp. 329-333.

[8] Y. Kubokawa, A general local ergodic theorem, Proc. Japan Acad. 48 (1972), pp. 461-465.

[9] - A local orgodic theorem for semi-group on $L_{p}$, Tôhoku Math. J. (2) 26 (1974), pp. 411-422.

[10] - Ergodic theorems for contraction semi-groups, J. Math. Soc. Japan 27 (1975), pp. 184-193.

[11] M. Lin, Semi groups of Markov operators, Boll. Un. Mat. Ital. (4) 6 (1972), pp. $40-44$.

[12] S. A. Mc Grath, On the local ergodic theorems of Krengel, Kubokawa, and Terrell, Comm. Math. Univ. Carolinae 17 (1976), pp. 49-59.

[13] D. S. Ornstein, The sums of iterates of a positive operator, in: Advances in Probability and Related Topics (edited by P. Ney), Vol. 2, pp. 85-115, New York 1970.

[14] R. Sato, Invariant measures for semigroups, Studia Math. 53 (1975), pp. 129-134.

[15] - A note on a local ergodic theorem, Comment. Math. Univ. Carolinae 16 (1975), pp. I-11.

[16] - On a local ergodic theorem, Studia Math. 58 (1976), pp. 1-5.

DEPARTMENT OF MATHEMATICS, JOSAI UNIVERSITY SAKADO, SATTAMA, JAPAN 